\title{
On Communication Protocols for Tactical Navigation Assistance
}

\author{
Evren Onem H. Birkan Yilmaz Fatih Alagöz Tuna Tugcu \\ Bogazici University \\ Department of Computer Engineering \\ Istanbul, Turkey \\ 0090-2123597686 \\ \{evren.onem, yilmhuse, alagoz, tugcu\}@boun.edu.tr
}

\begin{abstract}
Using tactical navigation systems is popular for military as well as search and rescue applications. For such applications, the significance of the tactical navigation systems arises especially when groups with the same goal move together on the terrain. Such systems rely on mobile ad-hoc communications rather than the classical infrastructure. In this paper, we aim to develop a cross-layer communication protocol suite for a tactical navigation assistance system. The traffic-adaptive medium access protocol (TRAMA) and a gossip-based ad hoc routing protocol are modified according to the needs of the tactical navigation applications. The efficiency of the MAC and the network layer is improved by using geographical information acquired by the application layer. Such an improvement relieves the MAC layer and the network layer from sending extra control messages, thereby leading to more efficient bandwidth usage. Furthermore, data fusion is performed for the frames in the MAC layer queue according to information quality. As a result, for environments with a low mobility rate, an efficient, collision-free MAC layer protocol and a fast data diffusing routing protocol is achieved.
\end{abstract}

\section{Categories and Subject Descriptors}

B.8.2 [Performance Analysis and Design Aids]: Performance evaluation of a communication protocol suite for tactical navigation assistance.

\section{General Terms}

Algorithms, Measurement, Performance, Design, Reliability.

\section{Keywords}

Tactical navigation, digital battlefield, cross layer, communication protocols, Gossip-based ad hoc routing, NGossip, C-TRAMAF.

\section{INTRODUCTION}

Traditionally, navigation applications have focused on positioning

Permission to make digital or hard copies of all or part of this work for personal or classroom use is granted without fee provided that copies are not made or distributed for profit or commercial advantage and that copies bear this notice and the full citation on the first page. To copy otherwise, to republish, to post on servers or to redistribute to lists, requires prior specific permission and/or a fee. MOBILWARE 2008, February 13-15, Innsbruck, Austria

Copyright (C) 2008 ICST 978-1-59593-984-5

DOI 10.4108/ICST.MOBILWARE2008.2874 and navigation to desired way points. However, treating navigation information as an aid for developing and maintaining situation awareness is an emerging trend. It has become evident that the availability and quality of digital positioning and navigation information at the individual platform level will play a key role not only in troop maneuvering, but also in cooperative movement of separate tactical elements.

For such systems, the geographical information of friendly and enemy forces should diffuse to the whole network as fast as possible. For efficient power utilization and low delay on message diffusion, a fine-tuned medium access protocol that provides collision-free access with high channel utilization is needed.

In this work, we aim to provide a cross-layer communication protocol suite for a Tactical Navigation Assistant (TNA). For the network layer, we define a new protocol that belongs to the 'Gossip' protocol family. For the medium access layer, we extend one of the sensor MAC protocols, TRAMA, in the sense that it performs data aggregation to form a frame with higher information quality. Moreover, both protocols use the application layer geographical information in order to prevent extra messaging in the original protocols.

The rest of the paper is organized as follows: In Section 2, we mention related work in the literature. In Section 3, we introduce the system architecture by discussing possible choices for both network and MAC layer protocols suitable for a tactical navigation system. We also describe our protocols for both layers. In Section 4, we discuss the results of a set of simulations conducted using OPNET network simulator. We finally reveal the conclusions and future directions in Section 5.

\section{RELATED WORK}

Previous work in the literature on tactical navigation consists of reliable and efficient communication protocols for Mobile Ad hoc Networks (MANETS) and mobility models for multi-hop networks.

In [1], Hong and his colleagues examined various mobility models in cellular and multi-hop networks. They introduced a new model and compared performances of different network protocols under their model.

Traffic analysis should be considered as a serious threat in enemy regions due to its feasible and intolerable risk prolificacy. Kong and Hong proposed Anonymous On-Demand Routing in [2], which is an on demand routing protocol that can be used in enemy 
regions. The protocol is based on the concept of broadcast with trapdoor information. Via route anonymity and location privacy, the protocol prevents adversaries from being able to trace network routes and the motion patterns in the network.

By examining the structure of communication networks in digital battlefield, some comparisons are performed in [3], and the needs of communication systems are emphasized by sample scenarios. The authors survey the characteristics of such networks and discuss the current directions for accomplishing such a digitization. They also describe the areas in which the civilian and military communication systems differ, and argue about a possible path for convergence of these two technologies.

Vogels and his colleagues describe some problems of scalable group communication systems and examine the epidemic techniques used for these problems in [4]. Authors also discuss the effects of gossip-based protocols on robustness.

Gossip-based ad hoc routing that can be combined with various optimizations is proposed in [5], where each node forwards a message with some probability. The protocol uses notably fewer messages than flooding and provides well-enough performance.

In [6], the traffic-adaptive medium access protocol (TRAMA) is introduced as an energy-efficient and collision-free MAC protocol. Since the protocol is specially designed for wireless sensor networks, it provides low-power synchronous channel access through the use of a slot assignment concept performed in a distributed manner.

\section{SYSTEM DESIGN}

In the design phase of a communication protocols suite, the most important concepts for a tactical navigation assistance system are low power operation and fast packet diffusion. In order to achieve the latter, we consider the family of gossiping protocols as our routing protocol candidates. However, we propose an extension to this family: the N-Gossip protocol. To achieve low power and efficient operation of the system we focus on modifying TRAMA, which is actually a sensor network MAC protocol.

\subsection{Application Layer}

A TNA is a handheld device that is capable of displaying the geographical information of friendly troops along with the enemy forces in a 3D navigation environment. Hence, the application layer for such a system heavily relies on fresh geographical data consisting of the real-world coordinates of all the troops in the related geographical area. Hence, the application layer packets of our tactical navigation system consist of the two dimensional coordinates of all the tactical navigation assistants and enemy forces on the terrain, along with a freshness value for each of the coordinates. Such a freshness value can be obtained by simply time-stamping each coordinate information in the packets.

In our proposed protocol, all the TNA devices over the terrain disseminate geographical information updates through the network in time periods of $\Delta t$ seconds. It should be noted that the application layer data can be veiled through the use of appropriate cryptographic techniques, such as constantly self-refreshing network-wide private keys implemented with hash-chains.

The enemy information is gathered either by the manual reporting of the troop members with TNA devices or through other supporting units such as a GEO, MEO, or LEO satellite subsystem, which can receive updates from the headquarters. Such a subsystem can also be used to improve the connectivity in the sense that whenever a TNA device does not have any neighbor, it sends its update packet to the supporting unit. Afterwards, the supporting unit relays that packet back to the network. However, such an operation can only be performed at the expense of high battery expenditure for that specific transmission.

\subsection{Network Layer}

Since one of our primary concerns is fast packet diffusion, using pure flooding at the routing layer is a solution. However, in this way many routing packets are propagated unnecessarily, which leads to unnecessary battery consumption. For this reason, we consider a gossiping-based approach, where each message is forwarded with some probability to reduce such an overhead. Unfortunately, this scheme still does not help much in its original format since in some executions the gossip fades away in a quick manner and hardly any node gets the message. In a tactical navigation system, any geographical update should quickly diffuse to the whole network, while keeping the battery expenditure as low as possible. Hence, we propose a new gossiping protocol, called $N$-Gossip, in which each node relays the packet exactly to $N$ of its neighbors or to all the neighbors if the number of its neighbors is less than $N$. In this way, the message does not die out if it does not get stuck at a dead-end. Thus, the network is not flooded with redundant packets as in pure flooding, provided that the protocol parameter $N$ is chosen appropriately.

The operation of N-Gossip protocol can be basically defined in the following sense: If a node executing this protocol has less than or equal to $N$ neighbors, it relays the packet to all of its neighbors. If it has more than $N$ neighbors, then it randomly chooses $N$ neighbors, and only relays to them. Every other node hearing the relayed packet acts in the same way.

\subsection{MAC Layer}

TRAMA is introduced for energy-efficient collision-free channel access in wireless sensor networks. TRAMA saves energy by ensuring that any unicast, multicast, and broadcast transmission occurs with no collisions while providing more than one transmission at the same time in diverse spatial locations. The protocol allows nodes to switch to a low-power, idle state whenever they are not transmitting or receiving.

TRAMA assumes that time is divided into slots and uses the result of a distributed calculation performed at each node in order to determine which node can transmit at a particular time slot. By using traffic information, the protocol allows nodes to determine when they can become idle. In the original protocol, each mobile device uses location information of its two-hop neighborhood by transmitting extra control packets and running localization algorithms. However, the application layer in our tactical navigation assistance system already possesses the location information of each node in the network. Accordingly, we drop the extra control messaging part of the original protocol and use the topology information provided by the application layer, which in turn leads to a cross layer protocol. By possessing the two-hop neighborhood location information and consequently assigning to each node distinct time slots, the hidden terminal problem is 
eliminated while taking advantage of the ability to perform multiple transmissions in different neighborhoods in the network. We extend TRAMA even further by performing data fusion if there are multiple packets in the queue when the MAC sublayer gains access to the medium. We use the concept of data fusion in order to aggregate the freshest geographical data into one unified packet and send only this single packet instead of sending multiple packets with partially fresh data. Though such an aggregation concept is not generally convenient for MAC layer, it is appropriate for our case due to the fact that the data diffusing through the network has no destination-specific properties. Instead, each packet has some geographical data with a certain degree of freshness. Hence, the overall freshness of the packet can be improved by the fusion process while relieving the node from trying to contend for the medium for all of the packets in its queue. Accordingly, the protocol reduces the number of transmissions performed by each node, which in turn reduces the energy consumption. Furthermore, since the protocol is aware of the distance to the destination node, transmission power is adjusted to a level that is slightly more than the needed amount. The excess part of the power is for the sake of guaranteeing the transmission success, since the real coordinates of the destination might be changed to some extent due to its mobility. We call the resulting protocol, the Cross-layer TRAMA with Data Fusion, as C-TRAMAF.

The lifetime is a major constraint in the concept of tactical navigation due to the fact that the state-of-the-art navigation handsets are powered by no ambient energy sources but battery. In a mobile ad hoc network, energy is wasted due to the following different types of overhead [7]:

Idle listening and overhearing: Because of the fact that there is no way for a mobile device to know when it should switch to receive mode, the radio must be kept active at all times. This fact leads to a key source of overhead since the amount of energy consumed in the receive mode is nearly equivalent to the transmit mode. However, C-TRAMAF is favored by a common sensor network phenomenon: the first transmitted bits of the MAC packet contain the destination address so that the neighbor nodes only have to listen to those first bits in order to understand whether the packet being transmitted is destined to them. As a result, nodes can save energy by immediately switching to sleep mode if the current packet is not destined to them.

Collisions: If multiple nodes transmit at a time and interfere with each other, such a situation leads to the waste of the energy that is spent to transmit and receive those packets. In C-TRAMAF, the access to medium is synchronized for the two-hop neighborhood. Therefore no collisions occur if the degree of mobility in the network is not in such a high level that the nodes in a neighborhood frequently join another neighborhood before the next slot assignment takes place.

Protocol overhead: The control packets used for discovering the neighborhood information do not contain useful information for the application itself, hence it is considered as overhead. However, C-TRAMAF is favored by the fact that the application layer itself has all the necessary geographical information that is fresh enough to be used in discovering the two-hop neighborhood. Such a special case relieves the protocol from performing extra control messaging.

\section{SIMULATIONS}

We have conducted a set of simulations using OPNET network simulator [8]. Our simulation parameters are given in Table 1.

Additionally, the battery expenditure model that we use is

$$
\text { trx_power_consumption }(\delta, \alpha, \beta, p)=\alpha \delta^{p}+\beta \text {, }
$$

where $\alpha=0.0001, \beta=0.1, \mathrm{p}=2.5$, and $\delta$ is the communication distance.

Table 1 - Simulation Parameters

\begin{tabular}{|ll|}
\hline Parameter & Value \\
\hline BER & 0.001 \\
\hline Reed-Solomon & $\mathrm{n}: 204, \mathrm{k}: 188$ \\
\hline Battery capacity & 1000 units \\
\hline Communication range & $150 \mathrm{~m}$ \\
\hline Time to live & 7 hops \\
\hline TNA device sleep time & $0.5 \mathrm{sec}($ duty cycle: $50 \%)$ \\
\hline \# transmission slots per cycle & $10 \mathrm{slots}$ \\
\hline Transmission slot length & $0.05 \mathrm{sec}$ \\
\hline Periodic update period $(\Delta \mathrm{t})$ & $1 \mathrm{sec}$ \\
\hline Mobility rate & $0-10 \mathrm{~km} / \mathrm{h}$ \\
\hline \# TNA devices & 32 \\
\hline \# enemy forces spotted & Up to 32 \\
\hline
\end{tabular}

We use a deterministic mobility model in which we define the trajectory according to a tactical scenario. We define a metric to assess the average staleness of the geographical data that the TNA devices in the network possess as

$$
\frac{\sum_{i=0} \sum_{j=0} \text { curr_time-geographical_freshness }(i, j)}{(\# T N A)^{2}}
$$

where geographical_freshness $(i, j)$ is the freshness of the information at the $i^{\text {th }}$ device about the geographical information of the $j^{\text {th }}$ device. Informally, this metric expresses the average age of the information in the network. Hence, by this metric we can judge the average quality of message diffusion in the network. A lower average staleness value means better message diffusion.

Our aim about the network layer is to investigate whether $\mathrm{N}$ Gossip protocol can produce geographical information in the network as fresh as pure flooding does. Since flooding is known to be the fastest diffusing protocol, it can be considered as a limit for providing the maximum level of freshness. We are also interested in the optimal choice of $N$, in order to reach the level of freshness that flooding achieves. For this reason, we vary the value of $N$ from 2 to a relatively large value for a digital battlefield, such as 16, which makes the protocol behave 
practically indistinguishable from pure flooding. Figure 1 depicts the average staleness values for N-Gossip protocol and pure flooding, which are taken from a sequence of experiments conducted using a mixture of nodes having zero, low, and medium degree of mobility according to our mobility model. As seen in the figure, on average, 4-Gossip exhibits a behavior very similar to pure flooding in terms of message diffusion. Such a result is obtained because of the fact that forwarding a message to more than 4 neighbors brings us no additional gain for improving the diffusion rate. However, it should be noted that limiting the number of receivers instead of pure flooding brings us the gain of light battery usage, while not sacrificing the high diffusion rate.

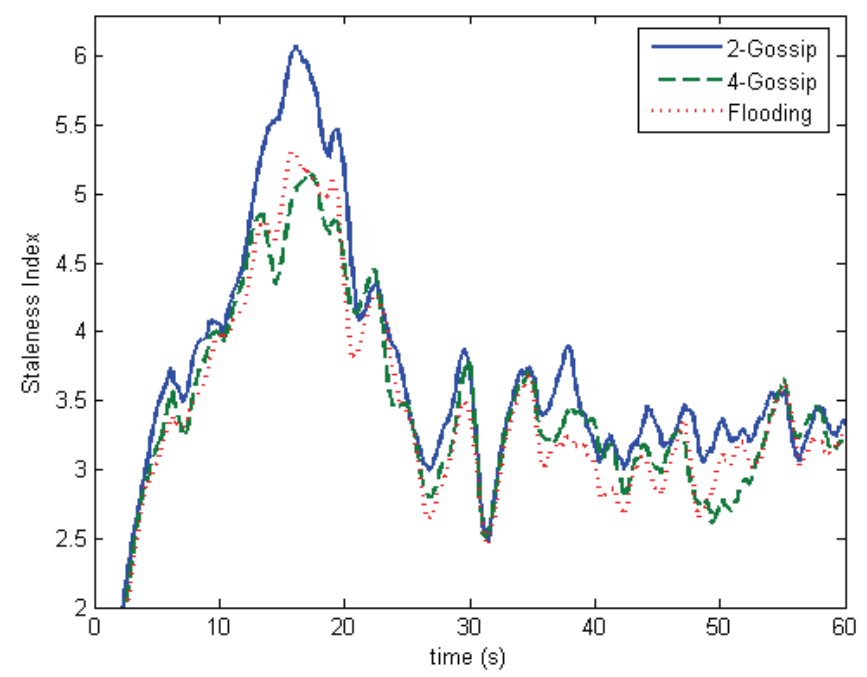

Figure 1 - Average staleness vs time for N-Gossip and Flooding with C-TRAMAF.

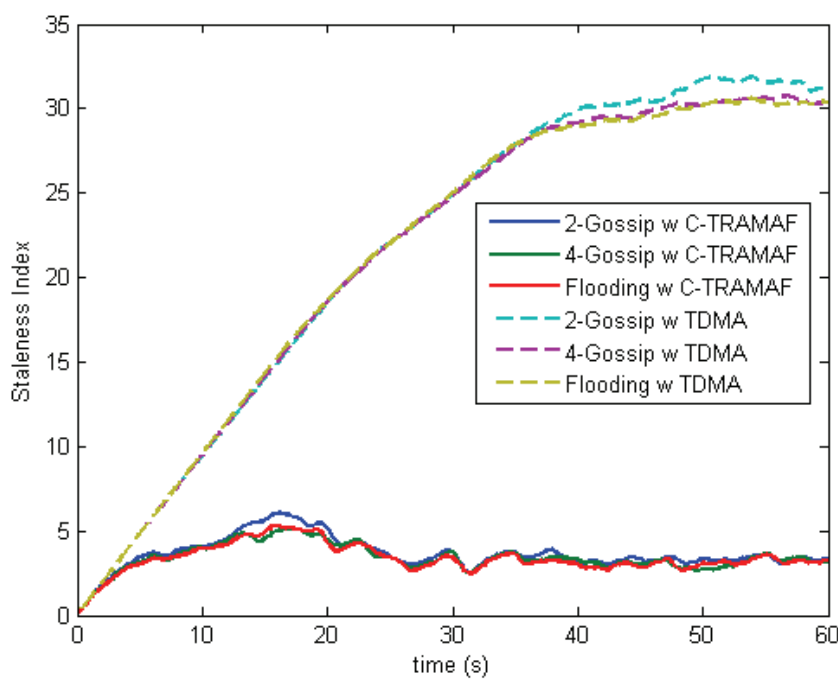

Figure 2 - Average staleness vs time for C-TRAMAF and TDMA.

Our aim concerning the MAC layer is to investigate whether CTRAMAF can outperform Time Division Multiple Access (TDMA), which is another synchronous medium access protocol that assures no collisions. Figure 2 shows average staleness values versus time for both C-TRAMAF and TDMA. It is apparent that C-TRAMAF outperforms TDMA in terms of message diffusion. Regardless of the routing protocol used in the network layer, CTRAMAF has an average staleness value of about 4 seconds, which does not fluctuate significantly. On the other hand, using TDMA may result in a very large staleness value implying a very slow message diffusion rate. We come up with such a result because a TDMA system allows only one device to transmit at a time, which in turn leads to a high overload in MAC layer queues. However, in C-TRAMAF, distant parts of the network can have successful transmissions at the same time. Furthermore, if there are multiple packets in the MAC layer queue at the time when the access to the medium is gained, C-TRAMAF uses data aggregation to merge all the packets in the queue to a single unified packet. This particular behavior greatly enhances the diffusion rate and improves the average freshness of the geographical information in the network.

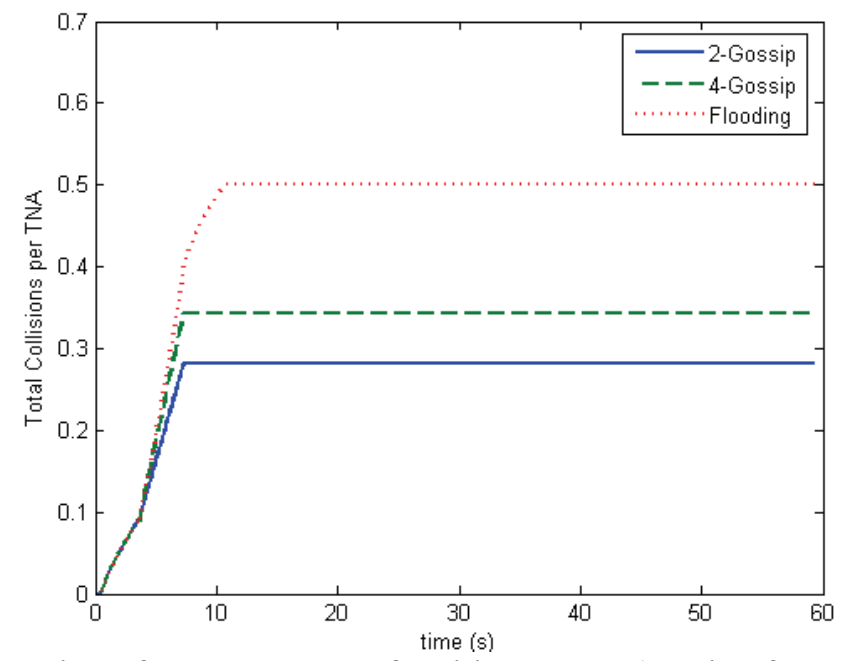

Figure 3 - Total number of collisions per TNA vs time for $\mathrm{N}$ Gossip and Flooding with C-TRAMAF.

In Figure 3, a specific simulation result is depicted where $\mathrm{x}$-axis represents time and $\mathrm{y}$-axis represents the total number of collisions per TNA device. In this scenario, there is a sharp decrease in the degree of mobility after the ninth second. We have three groups with different mobility patterns. One of the groups has no mobility, and the other two groups are mobile with a mobility level ranging in the interval $[0,10] \mathrm{km} / \mathrm{h}$. The reason for the increasing nature in the total number of collisions per TNA device in the first 11 seconds is the change in the topology due to high rate of mobility. A rapid change in the topology affects the contention period and leads to collisions due to the stale knowledge of two-hop neighborhood information. After the $11^{\text {th }}$ second, there is no more collisions in the network because of the fact that C-TRAMAF ensures a collision-free nature in the case of low mobility. The average number of collisions per TNA device has the highest value for the flooding case. It is apparent that when the number of destinations in the transmitted packet is increased, the total number of collisions per TNA device also increases. This is due to the fact that the probability of transmitting a packet in the same vicinity increases as well. 
In Figure 4, the lifetime comparisons for TDMA and C-TRAMAF are illustrated. N-Gossip protocols provide power saving up to $15 \%$ compared to flooding in both cases. This fact is due to two different reasons. The first reason is that if a node floods a packet to all its neighbors then it has to transmit the packet by using the amount of power required for the most distant neighbor. Therefore when N-Gossip is used, the required power to transmit to the chosen neighbors is less than or equal to the required power in the flooding case. The second reason is that if the number of destinations is increased then the branching factor of the same packet is also increased, which leads to more energy expenditure in the network.

We define the notion of lifetime as the time elapsed until the first node dies. It is depicted in the figure that when TDMA is used, the network lifetime is about $65 \%$ better than the case where $\mathrm{C}$ TRAMAF is used. This can be explained by the fact that the number of transmissions in one slot is exactly one for TDMA, whereas it is generally more than one for C-TRAMAF.

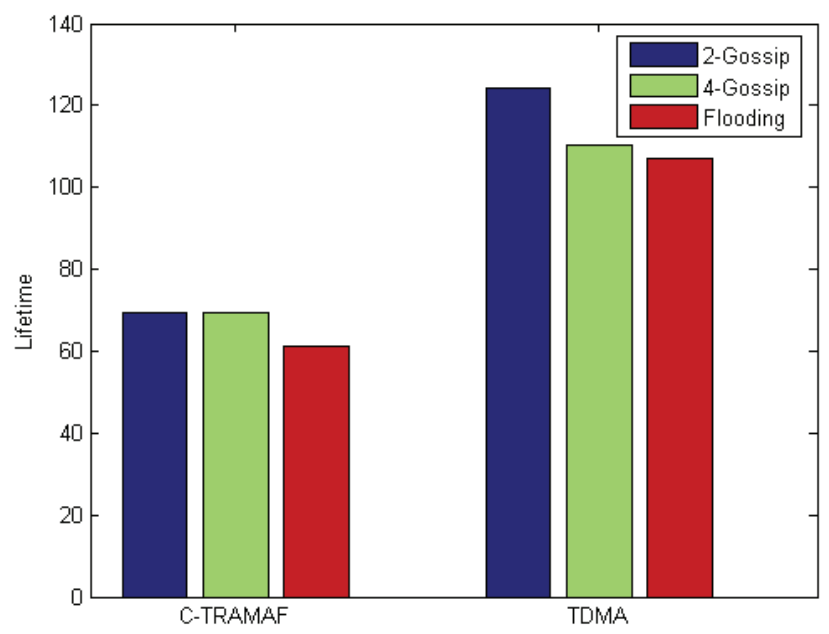

Figure 4 - Lifetime Comparisons for C-TRAMAF and TDMA

\section{CONCLUSIONS AND FUTURE DIRECTIONS}

Our simulations show that N-Gossip provides a high quality data dissemination that is similar to what is provided by flooding. Furthermore, the protocol ensures power savings up to $15 \%$ compared to flooding, by avoiding redundant packet transmissions. We also compare our MAC layer protocol CTRAMAF with TDMA, which is another collision-free synchronous MAC layer protocol. The results demonstrate that $\mathrm{C}$ TRAMAF outperforms TDMA in terms of providing fresher geographical knowledge within the network. Such a performance gain is provided by means of the data aggregation capability of our protocol that greatly reduces the queue size in MAC layer, while nevertheless granting fast message dissemination. However, because of the adequacy of multiple successful transmissions at a time slot, the lifetime of the whole network is diminished. Here, instead of the simple lifetime metric we use, using another metric that takes the utilization of the network into account could produce more reasonable results on this issue, which we can consider as future work. It should also be kept in mind that for tactical navigation purposes, fast data dissemination is a crucial property. Therefore, a low rate of message diffusion leading to a high level of staleness of the geographical knowledge can not be tolerated for the sake of providing a better lifetime. On the other hand, one should also be careful about choosing the optimal value for $N$, since an unnecessary increment in the value of $N$ can dramatically reduce the lifetime of the network while enhancing the data diffusion rate only by a little amount.

As future work, we should also consider a more inclusive framework by incorporating a wider range of mobility models, adding jammer models, considering a 3D terrain model with different densities of TNA devices, and performing BER vs FEC comparisons.

\section{ACKNOWLEDGMENTS}

This project is partially supported by Scientific and Technical Research Council of Turkey (TUBITAK) under grant number 104E032 and State Planning Organization of Turkey (DPT) under grant number 03K120250.

\section{REFERENCES}

[1] Hong, X., Gerla, M., Pei, G., and Chiang, C. 1999. A Group Mobility Model for ad Hoc Wireless Networks. In Proceedings of the 2nd ACM International Workshop on Modeling, Analysis and Simulation of Wireless and Mobile Systems, ACM Press, 53-60.

[2] Kong, J. and Hong, X. 2003. ANODR: Anonymous On Demand Routing With Untraceable Routes For Mobile Ad Hoc Networks. In Proceedings of the 4th ACM International Symposium on Mobile Ad Hoc Networking and Computing, ACM Press, 291-302.

[3] Sass, P. Communications Networks for the Force XXI Digitized Battlefield. Mob. Netw. Appl. 4, 3, 39-155.

[4] Vogels, W., van Renesse, R., and Birman, K. 2003. The power of epidemics: robust communication for large-scale distributed systems. SIGCOMM Comput. Commun. Rev., 33(1):131-135.

[5] Li, L., and Halpern, J., and Haas, Z. Gossip-based Ad Hoc Routing. 2006. IEEE/ACM Transactions on Networking, 479-491.

[6] Rajendran, V., Obraczka, K., and Garcia-Luna-Aceves, J. 2003. Energy-Efficient Collision-Free Medium Access Control for Wireless Sensor Networks. In Proceedings of the First International Conference On Embedded Networked Sensor Systems. ACM Press, 181-192.

[7] Langendoen, K., and Halkes, G. Energy-Efficient Medium Access Control. 2005. Book chapter in the Embedded Systems Handbook, CRC press.

[8] OPNET Network Simulator [Online]:http://www.opnet.com 